Macedonian Pharmaceutical Bulletin, 66 (Suppl 1) 139 - 140 (2020)

Online ISSN 1857 - 8969

UDC: $616.517-085$

DOI: 10.33320/maced.pharm.bull.2020.66.03.069

Short communication

\title{
Psoriasis therapy: Current state and future prospects
}

\author{
Beti Djurdjic $^{1,2 *}$, Vjeroslava Slavic $^{1,2}$ \\ ${ }^{1}$ Centre of Excellence for Biomedical Researches (CEBIMER), Institute for physical medicine, rehabilitation and \\ rheumatology "Dr Simo Milosevic", Sava Ilica 5, 85347 Igalo, Montenegro \\ ${ }^{2}$ Department of Pharmacy, Faculty of Medicine, University of Montenegro, Krusevac bb, 81000 Podgorica, Montenegro
}

\section{Introduction}

Psoriasis is a chronic inflammatory, immunemediated disorder that mainly affects the skin and joints. Psoriasis pathophysiology is characterized by abnormal keratinocyte proliferation and immune cell infiltration in the epidermis and dermis involving the innate and adaptive immune systems. Various types of psoriasis have been reported in literature and practice: plaque, guttate, inverse, pustular and erythrodermic psoriasis. Individuals with psoriasis are at an increased risk of developing other chronic and serious health diseases such as psoriatic arthritis, metabolic syndrome (or components of it), cardiovascular disorders, anxiety and depression, non-alcoholic fatty liver disease and Crohn's disease (Boehncke and Schön, 2015; Greb et al., 2016).

The aim of this paper was to review the existing therapy for psoriasis, as well as utilization of nanotechnology in formulation of drug delivery systems for psoriasis therapy.

\section{Current trends in treatment of psoriasis}

Psoriasis is a chronic relapsing disease, which often require a long-term therapy. The choice of therapy is determined by disease severity, comorbidities, and access to health care (Rendon and Schäkel, 2019). Current treatments for psoriasis include topical therapies, phototherapy, conventional systemic drugs and biologic agents. Most patients with psoriasis have mild to moderate disease that can be treated safely and effectively with topical therapies, including corticosteroids, vitamin D derivates (calcipotriol, calcitriol, tacalcitol), retinoids (tazarotene) tar, keratolytic agents (urea, salicylic acid), and emollients (Brandon et al., 2019). Results from a recent meta-analysis showed that a combination of corticosteroids and vitamin $\mathrm{D}_{3}$ was the most effective treatment for the scalp (Boehncke and Schön, 2015). Methotrexate (folic acid analogue that inhibits DNA synthesis by blocking thymidine and purine biosynthesis), cyclosporin ( $\mathrm{T}$ cellinhibiting immunosuppressant) and acitretin (retinoids) are systemic treatment options for psoriasis (Rendon and Schäkel, 2019). Over the past decade, several biologics have been developed and approved for the treatment of psoriasis. TNF- $\alpha$ inhibitors (etanercept, infliximab, adalimumab and certolizumab), first-generation biologics, are effective for plaque psoriasis and psoriatic arthritis. With the exception of etanercept, which is a fusion protein, other biologics are monoclonal antibodies. In the treatment of psoriasis, they show different PASI (Psoriasis Area Severity Index) score 75\% response rates: $52 \%, 59 \%, 80 \%$ and $83 \%$ for etanercept, adalimumab, infliximab and certolizumab, respectively (Rendon and Schäkel, 2019). Currently anti-IL-17 agents (secukinumab brodalumab, ixekizumab), anti-IL-23 inhibitors (guselkumab), and anti-IL-12-23 inhibitors (ustekinumab) are commonly used agents for plaque psoriasis and psoriatic arthritis (Brandon et al., 2019). In Cochrane Review (Sbidian et al., 2020) 140 studies were collected and analysed to compare the efficacy and safety of conventional systemic

\footnotetext{
* beti.djurdjic@gmail.com
} 
agents, small molecules, and biologics for people with moderate-to-severe psoriasis, and to provide a ranking of these treatments according to their eficacy and safety. Network meta-analysis at class level showed that all of the interventions were significantly more efective than placebo in terms of reaching PASI score $90 \%$. At class level, in terms of reaching PASI score 90 , the biologic treatments antiIL17, anti-IL12/23, anti-IL23 and anti-TNF- $\alpha$ were significantly more efective than the small molecules and the conventional systemic agents. The results showed that a selection of treatments from the class of biological medicines appear to be the most effective systemic medicines for achieving a $90 \%$ improvement in PASI score.

\section{Development of nanotechnology-based drug delivery systems for psoriasis therapy}

Psoriasis therapy based on conventional formulations can provide therapeutic benefits only to a limited extent (Rahman et al., 2014). Topical therapies have limitations as treatments have the potential to cause cutaneous side effects. While skin is the desired target of topical therapies, it is also a barrier to effective drug penetration and absorption, and the keratinocyte hyperproliferation associated with psoriasis further fortifies this barrier. While oral and injectable systemic therapies overcome the need to penetrate the stratum corneum, associated infection risk as well as liver, kidney, and bone marrow toxicities can limit their use in some patients (Murphy et al., 2019). Recent advancement in nanotechnology-based drug delivery systems has led to the possibility of improving the efficacy and safety of pharmacotherapeutic agents for psoriasis. Numerous studies in recent decade has demonstrated that nanoparticles as a drug carrier (such as liposomes, transfersomes, niosomes, polymersomes, nanoemulsions, solid lipid nanoparticles, polymeric nanoparticles, nanogels) can enhance the efficacy and reduce side effects of drug agents through increased skin retention and sustained drug release. The developed nanoemulsion loaded gel for topical co-delivery of clobetasol propionate and calcipotriol showed enhanced penetration and controlled release of drugs, increased anti-psoriatic efficacy compared to free drugs (Kaur et al, 2017). Kumar et al. (2016) created cyclosporine-loaded liposomes and incorporated these carriers into a Carbopol hydrogel. The clinical efficacy was studied in double-blind, randomized controlled trial of 38 patients with psoriasis. The developed formulation resulted in higher plaque clearance than a commercial cyclosporine cream but lower clearance than clobetasol propionate.

\section{Conclusion}

The results on animal models using nanoparticles via different route seem promising, but the number of studies in humans is limited and while the short-term use of nanocarriers appears safe, longterm outcomes are unknown. While recent research has demonstrated the benefit of nanotechnologybased drug delivery systems for psoriasis, more research is needed directed toward the clinical study to establish the fate of nanoparticles and optimize drug loaded nanocarriers for clinical use (Murphy et al., 2019; Rahman et al., 2014).

\section{References}

Boehncke, W.H., Schön, M.P., 2015. Psoriasis. Lancet 386, 983-94.

Brandon, A., Mufti, A., Sibbald, R.G., 2019. Diagnosis and management of cutaneous psoriasis: A Review, Advances in skin wound care 32(2), 58-69.

Greb, J.E., Goldminz, A.M., Elder, J.T., Lebwohl, M.G., Gladman, D.D., Wu, J.J., Mehta, N.N., Finlay, A.Y., Gottlieb, A.B., 2016. Psoriasis. Nat. Rev. Dis. Primers 2, 1-17.

Kaur, A., Katiyar, S.S., Jain, S., 2017. Nanoemulsion loaded gel for topical co-delivery of clobetasol propionate and calcipotriol in psoriasis. Nanomedicine 13, 1473-1482.

Kumar, R., Dogra, S., Amarji, B., Singh, B., Kumar, S.S., Vinay, K., Mahajan, R., Katare, O.P., 2016. Efficacy of novel topical liposomal formulation of cyclosporine in mild to moderate stable plaque psoriasis: a randomized clinical trial. JAMA Dermatol. 152, 807-15.

Murphy, E.C., Schaffter, S.W., Friedman, A.J., 2019. Nanotechnology for psoriasis therapy. Curr. Derm. Rep. 1-12.

Rahman, M., Akhter, S., Ahmad, J., Ahmad, M.Z., Beg, Z., Ahmad, F.J., 2014. Nanomedicine-based drug targeting for psoriasis: potentials and emerging trends in nanoscale pharmacotherapy. Expert Opin. Drug Deliv. 12(5), 1-18.

Rendon, A., Schäkel, K., 2019. Psoriasis pathogenesis and treatment. Int. J. Mol. Sci. 20(6), 1-28.

Sbidian, E., Chaimani, A., Afach, S., Doney, L., Dressler, K., Hua, C., Mazaud, C., Phan, C., Hughes, C., Riddle, D., Naldi, L., Garcia-Doval, I., Le Cleach, L., 2020. Systemic pharmacological treatments for chronic plaque psoriasis: a network meta-analysis. Cochrane Database of Systematic Reviews 1(1), 1-4.

Maced. Pharm. Bull. 66 (Suppl 1) 139 - 140 (2020) 